Behavior and Social Issues, 18, 58-80 (2009). (C) Todd A. Ward, Raymond L. Eastman, \& Chris Ninness. Readers of this article may copy it without the copyright owner's permission, if the author and publisher are acknowledged in the copy and the copy is used for educational, not-forprofit purposes.

\title{
An Experimental Analysis of Cultural Materialism: The Effects of Various Modes of Production ON RESOURCE SHARING
}

\author{
Todd A. Ward ${ }^{1}$ \\ University of Nevada, Reno \\ Raymond L. Eastman \\ Chris Ninness \\ Stephen F. Austin State University
}

\begin{abstract}
The 1980s witnessed an increased interest among behavior analysts regarding a paradigm in cultural anthropology known as cultural materialism. This perspective suggests that all behavior ultimately rests on the relationship between the natural environment and the methods used to obtain resources needed to maintain survival and a high standard of living, known as the mode of production. While useful, scientists in this area have overlooked one valuable resource-the laboratory. We manipulated the amount of resources dyads could harvest within blocks of five trials and across six conditions. Behavior consisted of harvesting and allocating resources. Token retention and a survival analogue were made contingent on resource sharing. Five of the seven dyads shared resources and no sharing occurred when participants could harvest sufficient resources to survive independently. Opportunities to integrate this research with optimal foraging research are discussed along with potential applications to realworld social issues.

KEYWORDS: cultural materialism, mode of production, resources, cooperation, token economy, analogue

The prediction and control of behavior drives behavior analytic theory (Skinner, 1953). As such, behavior analysts should consider ways to enhance the prediction and control of real-world behavior. One source of such enhancements could come from behavior analysts' increased interest in cultural anthropology (cf. Glenn, 1988, 2003; Lloyd, 1985; Malagodi, 1986; Malagodi \& Jackson, 1989; Malott, 1988; Vargas, 1985). The present study seeks to begin the development of
\end{abstract}

\footnotetext{
${ }^{1}$ The current study comprised part of a Master's thesis conducted by the first author at Stephen F. Austin State University in Nacogdoches, TX, in conjunction with the departments of psychology and human services. Author contact: wardt14@unr.nevada.edu.
} 


\section{MODES OF PRODUCTION}

an experimental methodology to investigate relations between behavior analysis and cultural anthropology.

\section{Behavior Analysis ANd CUltural MATERialism}

In their research, behavior analysts typically examine behavior-environment relations while assuming or "holding constant" survival contingencies. Marvin Harris's (1979) cultural anthropological paradigm known as cultural materialism, on the other hand, examines the behavior of human populations relative to survival as a dynamic variable. While behavior analysts assume survival in their research, cultural materialists do not.

Before the essential concepts of cultural materialism are discussed, consider the following. One could conclude that behavior-environment relations are nested within a culture whose characteristics are largely influenced by the means in which the members of the culture acquire the resources needed for survival. Cultural materialism could benefit behavior analysis by examining the effects of survival on behavior in greater detail, thus potentially increasing the prediction and control of behavior to unknown levels. Behavior analysis, in turn, could benefit cultural materialism by exposing its concepts to what may be the most rigorous and practical experimental methodology in all of the behavioral sciences (cf. Sidman, 1960; Skinner, 1953).

\section{Cultural Materialism: Essential Concepts}

Cultural materialists posit that the interaction between the mode of production (i.e., the means by which the members of a culture obtain resources, such as farming or various types of employment) and the mode of reproduction (i.e., the means by which the members of a culture influence population growth, such as mating patterns and nutrition) form the primary impetus in cultural development (Harris, 1979). Stated briefly, an ever-expanding population requires production intensification (i.e., working to produce more food, water, and energy), which temporarily raises the standard of living (i.e., brings more reinforcers into the environment), but eventually requires further intensification to accommodate the larger population, and simultaneously depletes the surrounding resources (Harris, 1977, 1979). According to Harris (1979) such intensification/depletion cycles then probabilistically determine the rest of a population's interactions, which is divided into a tripartite scheme: the (a) infrastructure (i.e., the mode of production and reproduction), (b) structure (i.e., behavior relevant to orderliness among populations, such as family structure, political organizations, and police), and (c) superstructure (i.e., the "recreational, 
sportive, and aesthetic" (p. 52) aspects of behavior, such as art, science, and advertising).

\section{Production EFFiciency ANd SHARING AMONg Hunter-Gatherers: A Negative Correlation}

The following example of economic egalitarianism in hunter-gatherer societies briefly illustrates what Glenn (1988) calls "infrastructural contingencies" (p. 171), or how modes of production and reproduction can affect a population and, in particular, the probability of sharing resources (e.g., giving food to one another). The reader will note an apparent negative correlation between production efficiency and resource sharing.

Harris (1979) accounted for the evolution of our social world starting with hunter-gatherers' reliance on animal migratory patterns which set the conditions for a relatively mobile existence, as well as economic egalitarianism. In huntergatherer cultures, the probability of any individual having a successful hunt was relatively low, whereas the probability of any group member having a successful hunt was relatively high. Thus, while any one individual hunter may have been incapable of surviving or generating a high standard of living, members of the group relying on each other for resources when others came up short ensured everyone's survival and standard of living. Having a mobile existence also led to highly mobile structures (e.g., housing) which mitigated two conditions: (a) the number of disputes between groups (i.e., disputing parties could simply move to another location), and (b) any hierarchical class-type structure. The high protein/low carbohydrate diet also decreased fertility in these groups, which slowed population growth, thereby reducing demand for resources.

The preceding example seems to describe a negative correlation between production efficiency and resource sharing. The present study sought to create a simplified experimental analogue to investigate a few possible aspects of this complex relationship within the framework of Harris's before mentioned (1977, 1979) intensification/depletion cycles, which seem to be an inherent property of resource-population relations. Two aspects of resource production were manipulated in the present study: (a) the percentage of trials with resources available for harvest, and (b) within that percentage, the range of possible resources made available for harvest. These results may contribute to an increased understanding of some specific features of production efficiency that affect resource sharing.

In following Harris's $(1977,1979)$ proposition that all behavior depends first on acquiring resources, and Skinner's (1981) proposition that behavior depends 


\section{MODES OF PRODUCTION}

on a behaving organism, which requires resources to survive, all behavior in this study consists of allocating resources in a laboratory analogue procedure.

Participants in the current study were required to harvest functional, laboratory-based resources and to meet analogues to survival contingencies before any extra resources could be allocated towards tokens. Token retention in this study can be seen as a laboratory "standard of living." If a participant's survival contingencies were not met then he/she could not continue and lost all tokens earned in the current condition as well as any stored resources. Sometimes, any one participant could not meet his/her survival contingency individually. Thus, under certain conditions, if any one participant wanted to survive and retain tokens, both dyad members would have to share resources.

\section{EXPERIMENT 1}

\section{Method}

\section{Participants}

Six females and two males, who comprised four dyads, participated for class credit and had a chance to win a \$25 gas card. The participants were aged 18-21 with the exception of one participant, age 43. Dyads 1 and 3 contained all females, while Dyads 2 and 4 each contained a male and female. The age difference within each dyad was within four years, with the exception of Dyad 4 which contained a 20-year-old female and a 43-year-old male. All of the participants were Caucasian undergraduates from a mid-sized Texas university, with the exception of the 43-year-old male who was Hispanic. All of the participants were recruited through an online sign-up system maintained by the psychology department. Before a participant decided to sign-up for the study, they were presented with a statement informing them of a chance to win a $\$ 25$ gas card.

The research assistant (hereafter referred to as "experimenter") was a 27year-old female graduate student. Her duties were to administer the experiment through verbal prompts (i.e., saying "remove all your sticks from the participation requirement," "remove all your (plain/colored) sticks from the store," and "draw a card") and nonverbal actions (e.g., handling the condition decks, presenting and removing tokens). 


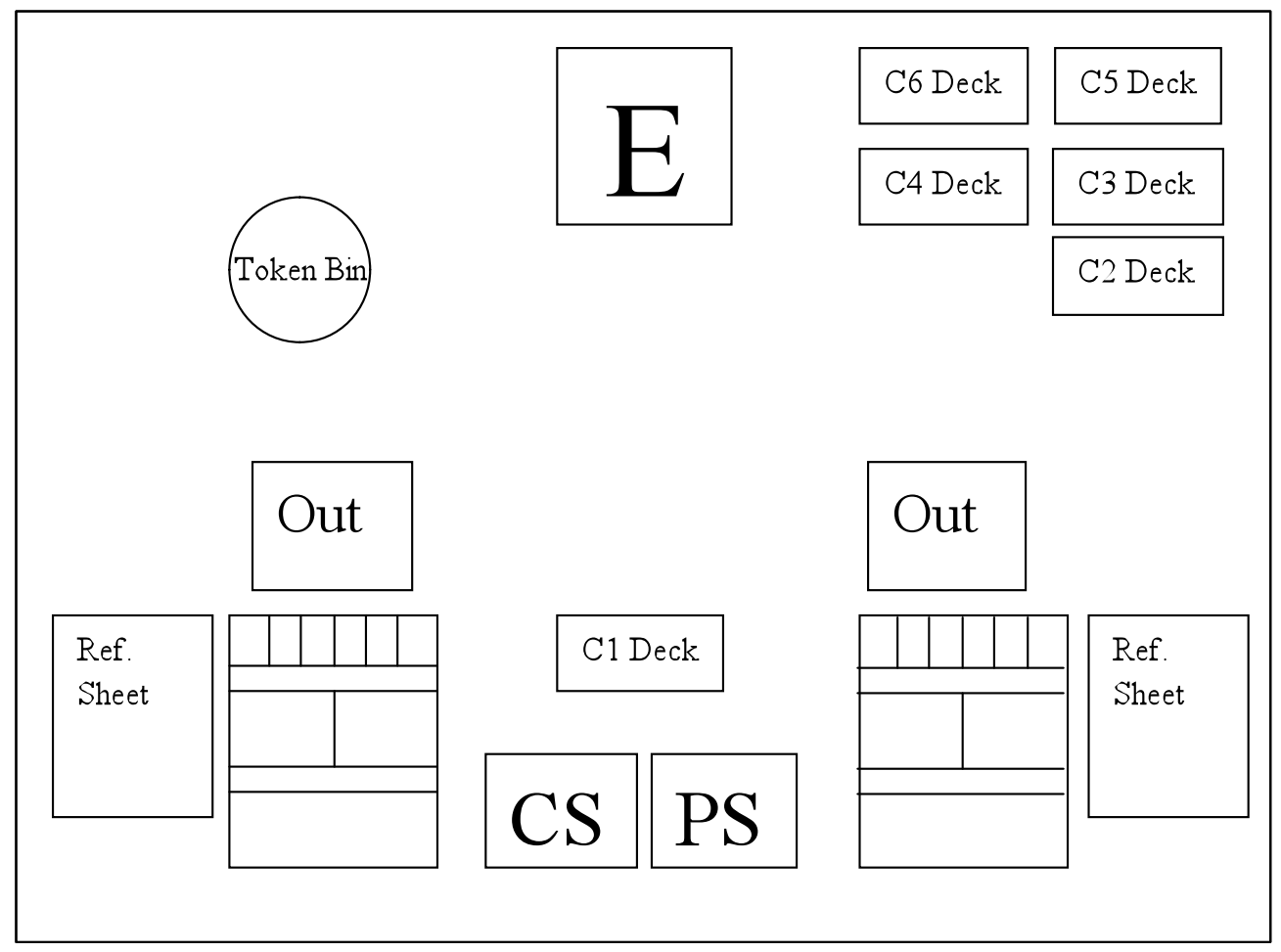

Figure 1. The laboratory setup for each dyad.

\section{Apparatus}

Figure 1 illustrates the laboratory arrangement in which two participants, each with their own 14"x23" foam board (see Figure 2), sat across from the experimenter, denoted "E." Each board was divided into various areas in which to allocate colored and plain wooden craft sticks drawn from bins denoted by "CS" for "colored sticks" and "PS" for "plain sticks" in the figure. Since all experimental behavior depended on having these sticks, the sticks were functionally analogous to resources and will be referred to as resources for the remainder of this paper.

To simulate reinforcement characterized by the term "standard of living," plastic beads served as tokens, which were earned based on the allocation of resources. Six stacks of notecards (one stack for each condition) were used in this study (see Figure 3). A participant would draw a card from the top of the deck, which would inform the dyad of the kind (i.e., colored or plain) and quantity of resources to be obtained each trial. 


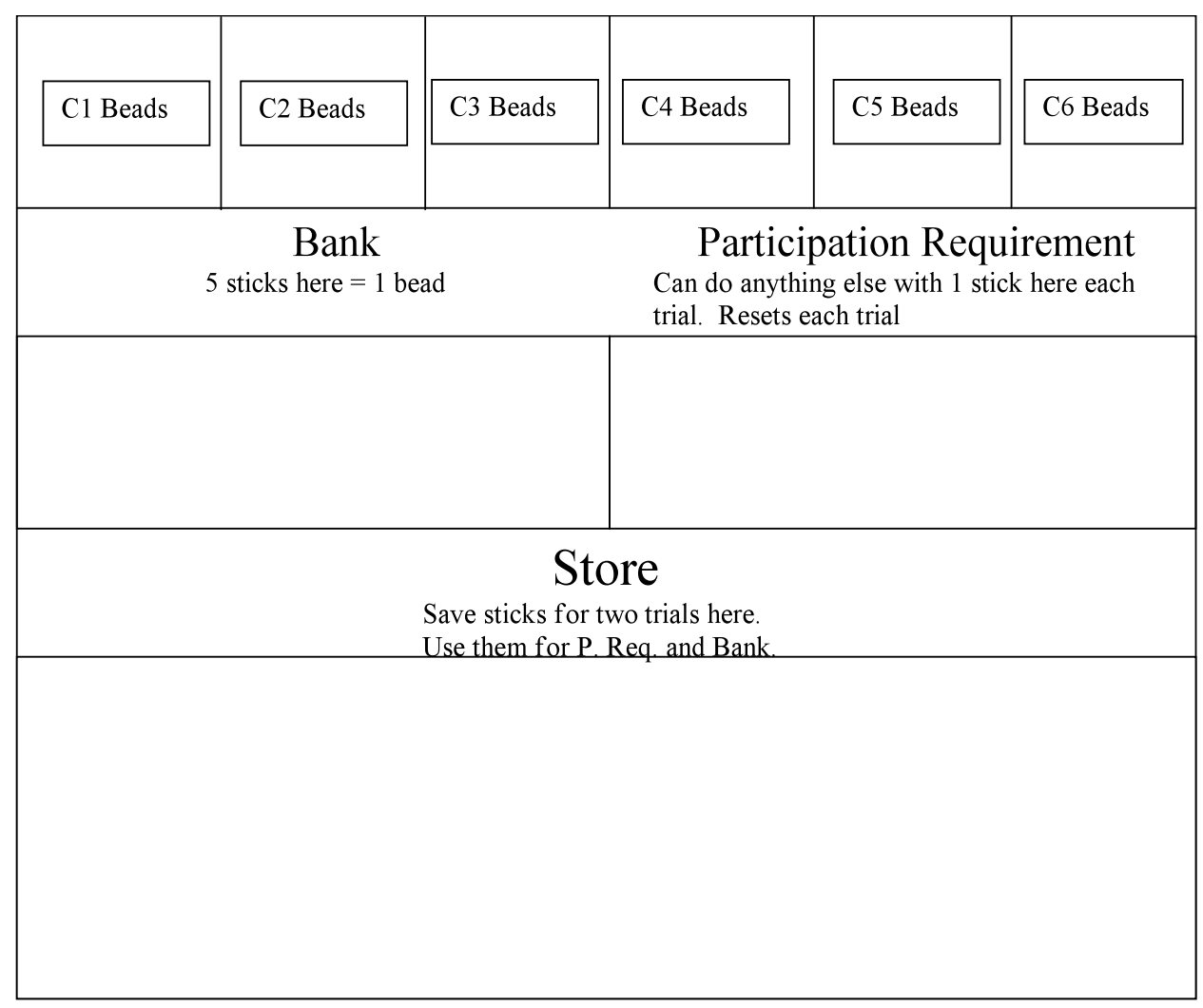

Figure 2. The apparatus for each individual participant.

A sheet of paper displaying the word "Out" was placed on a participant's board when he/she ran out of resources. Each participant had before them a reference sheet, which provided a set of rules to keep them aware of the experimental contingencies (see Appendix A). All sessions were videotaped and observed by the first author from an adjacent room through a two-way mirror, which reduced the experimenter's data-recording tasks during the study to tracking the trial-blocks and condition-change criteria.

\section{Design}

This study incorporated six conditions, each of which denoted different probabilities of resource obtainment. Condition 1 served as a baseline condition in 


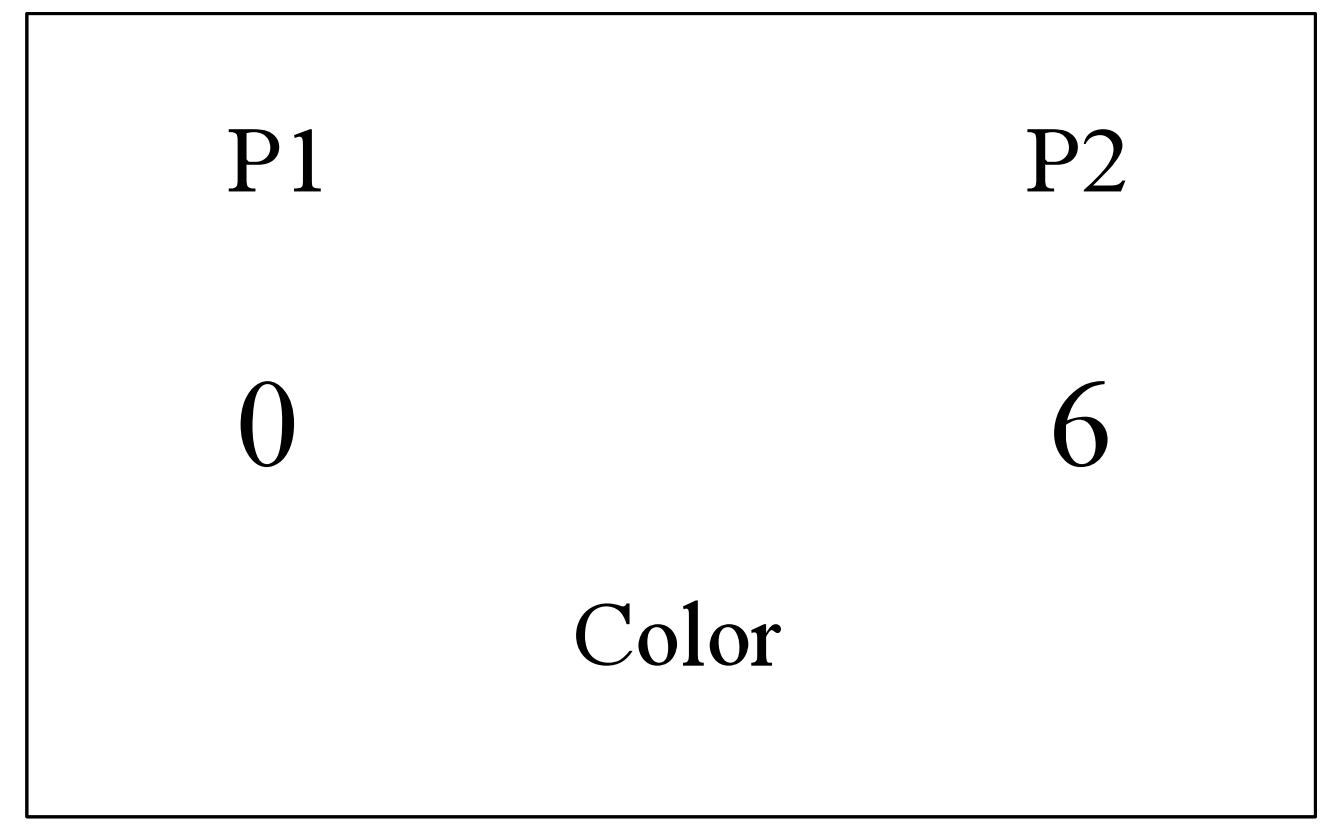

Figure 3. A sample notecard drawn by a participant at the beginning of each trial.

which both participants could harvest and allocate resources independently, while Condition 2 depleted the probability of resource obtainment such that neither participant could earn tokens at all. Conditions 3-6 were characterized by a progressive intensification in resource availability, thereby steadily increasing the probability of resource obtainment. Sample trial blocks for each condition are shown in Figure 4.

The sequence of conditions were designed based on Harris's $(1977,1979)$ discussion of intensification/depletion cycles, which presumably function to propel cultural change, and seem to be an inherent property of resource-behavior relations. As such, the sequence of conditions was held constant across all dyads (i.e., sequence effects in this study were intentionally confounded). That said, whether or not a "culture" is present in this study is irrelevant to the goals of the study, which revolve around an experimental analysis of modes of production on elementary behavioral phenomena. 


\section{MODES OF PRODUCTION}

Condition 1: Baseline-Intensive Mode of Production

Participants could ensure survival and earn tokens individually.

Expected No Sharing

Trials with Available Resources: 5/5

Possible Resources when available: 2

\begin{tabular}{lll} 
& P1 & P2 \\
\hline 1) & 2 & 2 \\
2) & 2 & 2 \\
$3)$ & 2 & 2 \\
$4)$ & 2 & 2 \\
5) & 2 & 2 \\
\hline
\end{tabular}

Condition 2: Depleted Model of Production

Participants could not retain tokens regardless of actions.

Trials with Available Resources: 2/5

Possible Resources when Available: 2

\begin{tabular}{lll} 
& $\mathrm{P} 1$ & $\mathrm{P} 2$ \\
\hline 1) & 0 & 2 \\
2) & 2 & 0 \\
$3)$ & 2 & 0 \\
$4)$ & 0 & 0 \\
5) & 0 & 2 \\
\hline
\end{tabular}

Conditions 3-6: Gradually Intensifying Modes of Production

Sharing was expected to increase until sufficient resources were harvested individually, then decrease.

Trials with Available Resources: $2 / 5$

Possible Resources when Available: 2-3, 2-4, 2-5, 2-6

\begin{tabular}{cccccccccccc}
\multicolumn{3}{c}{ Condition 3 } & \multicolumn{3}{c}{ Condition 4 } & \multicolumn{3}{c}{ Condition 5 } & \multicolumn{3}{c}{ Condition 6} \\
\hline & P1 & P2 & & P1 & P2 & & P1 & P2 & & P1 & P2 \\
\hline 1) & 3 & 2 & 1) & 0 & 2 & 1) & 5 & 0 & $1)$ & 0 & 0 \\
2) & 0 & 0 & $2)$ & 4 & 0 & $2)$ & 0 & 4 & $2)$ & 2 & 6 \\
$3)$ & 0 & 0 & $3)$ & 3 & 2 & $3)$ & 0 & 3 & $3)$ & 0 & 0 \\
$4)$ & 0 & 3 & $4)$ & 0 & 0 & $4)$ & 2 & 0 & $4)$ & 2 & 0 \\
$5)$ & 2 & 0 & $5)$ & 0 & 0 & $5)$ & 0 & 0 & $5)$ & 0 & 6 \\
\hline
\end{tabular}

Figure 4. Sample modes of production for one trial block in each condition. 


\section{Procedure}

After the participants signed the informed consent, the experimenter read aloud the instructions (see Appendix B) regarding the workings of the experimental environment. The participants subsequently followed along with the abbreviated instructions on the reference sheets. The experiment began with the experimenter saying "draw a card" and one dyad member would draw a card from the condition deck. The card informed each participant how many and what kind of resources (i.e., colored or plain) they would each harvest for that trial. Each participant then selected the quantity and kind listed under "P1" for Participant 1 or "P2" for Participant 2 on the card. Participants could then allocate resources in the four ways discussed below.

Survival and sharing. Above all else, each participant had to satisfy a survival contingency that allowed them to continue through the trial. This was satisfied by allocating one resource to the box labeled "participation requirement" at the beginning of every trial (see Figure 2). Once this was satisfied, participants could allocate any extra resources in various ways including toward the receipt of tokens. If a participant failed to meet this requirement, he/she could not continue, was marked as "out," lost any stored resources and all tokens earned in the current condition, and could not do anything until the other participant similarly failed to ensure his/her own survival. If both participants failed to survive, the dyad could restart within the current condition in an effort to learn how to prevent the loss of tokens.

Under conditions of inefficient modes of production, a participant could not survive and retain tokens on his/her own. Thus, the only way for either participant to survive and retain tokens until the end of the experiment would be for both participants to share resources (i.e., giving resources to a partner). Thus, we hypothesized that conditions of resource scarcity would evoke resource sharing analogous to Harris's (1979) description of hunter-gatherers. It should also be noted that the relation between sharing and token retention was not explained to the participants.

Earning tokens. After a participant ensured his/her own survival for a trial, extra resources could be allocated to his/her "bank" (see Figure 2). Allocating resources to the bank was analogous to spending money in the real-world and, therefore, could not be retrieved once allocated. For every five resources a participant allocated to his/her bank, he/she received one token (i.e., an FR 5 token economy). A participant's name was then entered into a drawing for a \$25 gas card for every token he/she retained throughout the entire experiment.

Storing. Participants could also store resources for use on the subsequent trial by allocating them to the "store" (see Figure 2). In other words, a participant may 


\section{MODES OF PRODUCTION}

receive resources and allocate some of them to the store on trial 1, use the stored resources on trial 2, and any resources earned on trial 1 still left in the store upon the initiation of trial 3 would expire (i.e., were taken away by the experimenter at the beginning of trial 3 such that they could not be used by the participant). Allocating resources to the store was analogous to any real-world resource preservation activity such as storing milk in the refrigerator to prevent spoiling. Storing would presumably increase under inefficient modes of production. Storing required the experimenter to keep track of resource age. Thus, two types of resources were used (i.e., colored and plain) and each trial alternated between a "color trial" and a "plain trial." At the beginning of every trial, before the experimenter would say "draw a card," the experimenter would say "remove all (colored/plain) sticks from your stores" depending on the trial. Thus, resources became unusable at the beginning of the second trial after they were received.

\section{Experimental Conditions}

As stated previously, this study had six conditions that were modeled after Harris's (1979) description of intensification/depletion cycles. Since these cycles were presumed to be inherent in resource-behavior relations, each dyad experienced the same sequence of conditions and each dyad experienced the same availabilities of resources, samples of which are shown in Figure 4. The first condition was the baseline condition because the participants would be harvesting sufficient resources per trial to fulfill his/her own participation requirement and allocate his/her own resources toward tokens each trial. During this condition, each participant received two resources each trial. The experimenters hypothesized that each participant would allocate one resource to the participation requirement and one to the bank each trial in this condition (see Figure 4). After a participant received six tokens or a dyad underwent six restarts, the next condition commenced.

While the first condition represented a mode of production that enabled a steady reinforcement rate, the second condition represented a sudden depletion of resources with the addition of zero-trials. In this condition, each participant still received two resources, but only during two out of every five trials. The remaining three out of every five trials resulted in zero resources. The specific two trials within each 5-trial block in which resources were harvested were randomly determined before data-collection commenced and were identical for every dyad in the current study. Thus, no tokens could be retained in Condition 2 regardless of the participants' actions because the dyads experienced multiple restarts in which all tokens and stored resources were eliminated; the participants would retain the tokens earned only during the first condition. 
After six restarts in Condition 2, Condition 3 commenced. As shown in Figure 4, for Condition 3, participants still received resources on every two out of five trials. However, the range of possible resources obtainable during these trials increased by one. Thus, on every two out of five trials a participant would harvest either two or three resources. The remaining conditions simply increased the range of harvested resources by one. Thus, in Condition 4 the range of resources was 2-4, in Condition 5 the range was 2-5, and in Condition 6 the range was 2-6. At the end of the entire experiment, the participants were debriefed on the purpose of the experiment and completed a demographics form.

\section{Results}

The consequences of resource allocation observed in Experiment 1 were: (a) the predicted "group-saved" consequence, whereby the recipient of a shared resource remained in the trial due to the fulfillment of the participation requirement from the shared resource, and (b) "recipient-more," whereby the recipient of shared resources ended up with more resources on his/her board than the giver. While the antecedent conditions occasioning sharing were unclear for recipient-more, sharing that produced the group-saved consequence was always occasioned by the recipient first being out of resources.

The results of Experiment 1 are shown in Figure 5, which omits data for Dyad 4 due to zero sharing responses observed during the experiment. Although three of the four dyads shared, no dyad retained any tokens upon completion of Conditions 2-6. In other words, the participants never identified how to avoid the loss of tokens. Nevertheless, giving occurred anyway. An explanation to this finding will be giving in the General Discussion.

All dyads responded individually during Condition 1 as predicted. For Dyad 1, sharing occurred once in Conditions 5 and 6. During the first instance of sharing, the recipient incorrectly allocated the newly received resource towards the partner's tokens (i.e., the partner's bank) instead of survival (i.e., the partner's participation requirement) and was, therefore, "out." During the second instance, however, the recipient correctly maintained the participation requirement and, therefore, kept participating for that trial. Thus, the second instance of sharing functioned to save the group.

Dyads 2 and 3 generated sharing that produced an unpredicted recipient-more consequence. For Dyad 2, sharing occurred twice in Condition 5, while a third occurred in Condition 6. Two instances of sharing, one in Condition 5 and the other in Condition 6, produced both group-saved and recipient-more consequences simultaneously. Thus, two data points in each of these conditions 


\section{MODES OF PRODUCTION}

\section{Dyad 1}

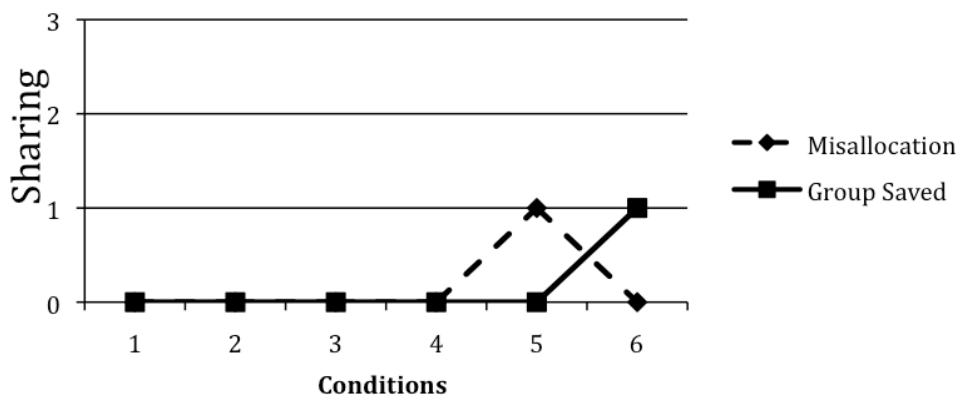

\section{Dyad 2}

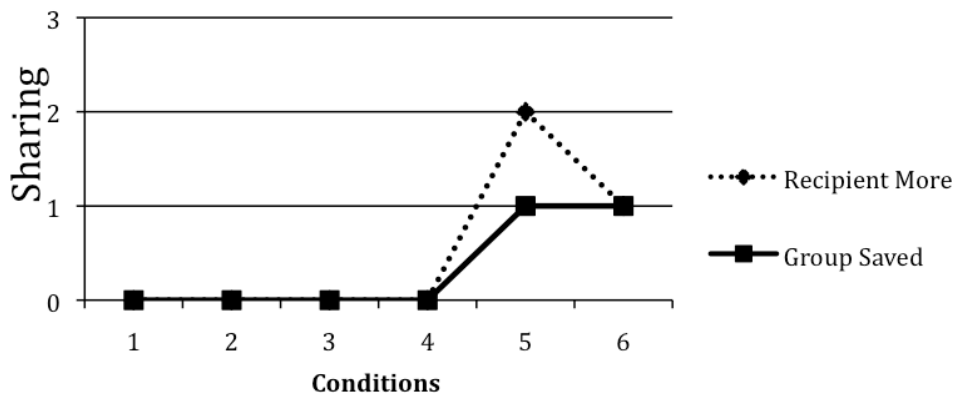

Dyad 3

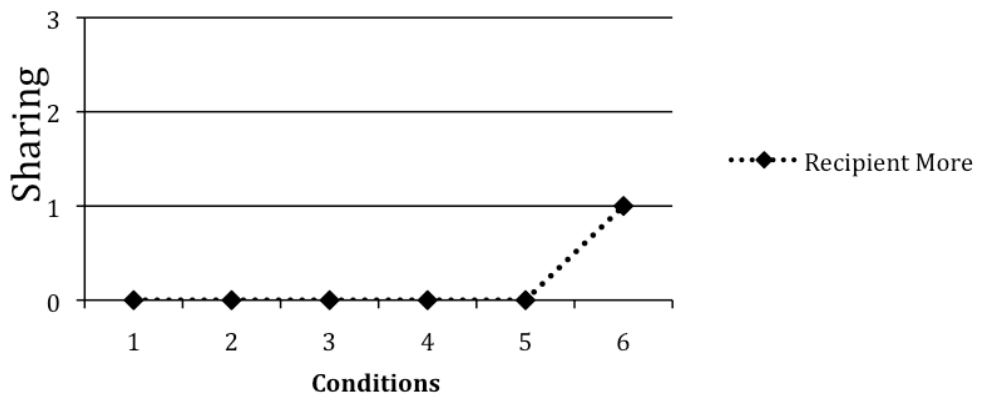

Figure 5. Instances of sharing per condition in Experiment 1 for Dyads 1, 2, and 3. Dyad 4 had no sharing data. For Dyad 2, two data-points in Condition 5 and two in Condition 6 each refer to two consequences generated by a single instance of sharing. 
refer to the same instance of sharing. Dyad 3 shared once in Condition 6 which produced a recipient-more consequence.

The rates of sharing observed in Experiment 1 were relatively low. The possibility arose that perhaps the experimental arrangement was an inaccurate model of the natural environment. In hunter/gatherer terms, if one runs out of resources, he or she dies and loses everything. In less extreme, more monetary, circumstances, if one loses one's job and runs out of money, one has to start over from zero. It was hypothesized that removing the token retention feature, whereby participants could keep all tokens earned during previous conditions when "restarts" occur, would increase the aversiveness of acting individually and evoke higher rates of sharing. Experiment 2 was designed to test this hypothesis.

\section{EXPERIMENT 2}

\section{Method}

\section{Participants}

Five females and one male, who comprised three dyads (i.e., Dyads 5, 6, and 7), participated for class credit and had a chance to win a $\$ 25$ gas card. The participants were aged 18-21 with the exception of one participant, age 30. Dyads 5 and 6 contained all females, while Dyad 7 contained a male and a female. The age difference within each dyad was within one year, with the exception of Dyad 5 which contained a 21-year-old female and a 30-year-old female. All of the participants were undergraduates, three were Caucasian and one AfricanAmerican, and two participants declined to identify their ethnicity. No participants from Experiment 1 participated in the current experiment.

\section{Apparatus}

The reference sheets and the instructions were altered slightly to state that a failure to maintain the participation requirement resulted in the total loss of tokens.

\section{Design}

The design was identical to that used in Experiment 1.

\section{Procedure}

The procedure was identical to that used in Experiment 1, with the exception that restarts resulted in, not only the loss of all tokens and resources earned during 


\section{MODES OF PRODUCTION}

the current condition, but the loss of all tokens earned throughout the entire experiment.

\section{Results}

The results for Experiment 2, shown in Figure 6, were analyzed in terms of sharing per condition and the immediate consequences resulting from each instance of sharing. No sharing was observed in Dyad 6. As in Experiment 1, no dyad retained any tokens in Conditions 2-6 and, due to the new manipulation, no dyad in Experiment 2 retained any tokens at all.

Figure 6. Instances of sharing per condition in Experiment 2 for Dyads 5 and 7. Dyad 6 had no sharing data. For Dyad 5, two data-points in Condition 4 and two in Condition 5 each refer to two consequences generated by a single instance of sharing.

\section{Dyad 5}

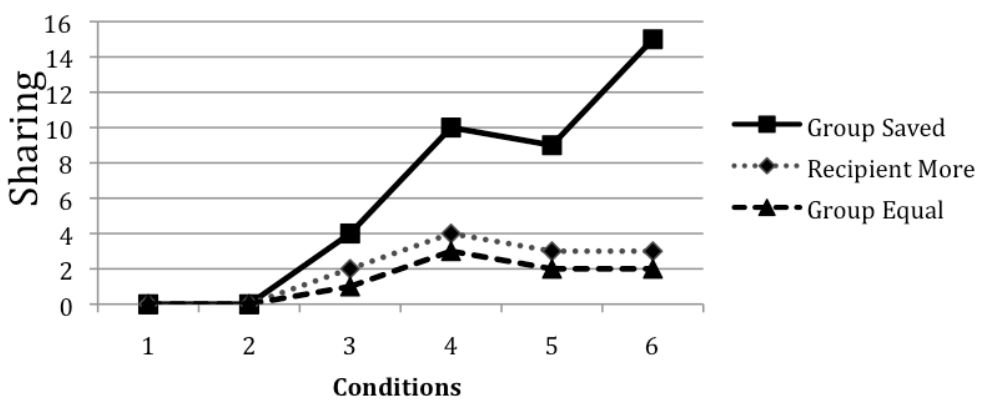

Dyad 7

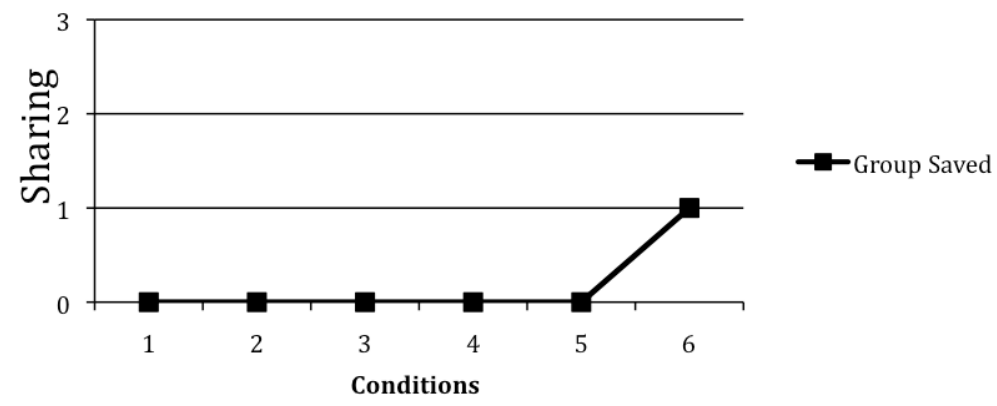


Dyad 5 generated the highest rate of sharing out of all the dyads in the entire study. Sharing first appeared in Condition 3 and increased in rate throughout the rest of the conditions. Sharing that produced group-saved consequences were most frequent, followed by recipient-more and the appearance of a novel "groupequal" consequence. The group-equal consequence was characterized by both participants having an equal number of resources immediately after sharing. The antecedent conditions that occasioned group-equal consequences were unclear. Two instances of sharing in Dyad 5 generated multiple consequences: one in Condition 4 generated a group-saved and a group-equal consequence while another in Condition 5 generated a group-saved and a recipient-more consequence. Thus, two data points in both of these conditions refer to the same instance of sharing. Dyad 7 shared once in Condition 6, which produced a groupsaved consequence.

\section{GENERAL DiscuSSION}

In this study, we manipulated: (a) the percentage of trials with available resources, and (b) the range of resources made available during these trials. Subsequently, we observed that sharing never occurred during the first condition where each participant could ensure his/her own survival and earn tokens independently. We also observed the emergence of sharing in 5 out of 7 dyads, which occurred in conditions 3-6. As seen in Figures 5 and 6, within the dyads that shared, four had similar rates of sharing, two of which began in Condition 5 (Dyads 1 and 2) while the other two began in Condition 6 (Dyads 3 and 7). The remaining dyad (Dyad 5) displayed strikingly high rates of sharing that began in Condition 3 and increased through the rest of the experiment. While the highperformance of Dyad 5 is unique to Experiment 2, with the removal of the tokenretainment feature, the finding is not robust enough to warrant clear conclusions regarding this between-experiment manipulation.

\section{Limitations and Suggestions for Future Research}

The current study carried with it certain limitations attributable to the exploratory nature of the investigation. First, one must be cautious when drawing conclusions regarding functional relations presumed to account for the data in this study. The three so-called consequences in which the data were organized (i.e., "group-saved," "recipient-more," and "group-equal") are merely descriptive and based solely in terms of temporal contiguity with sharing responses. Thus, the stimulus functions of these apparent consequences were not demonstrated experimentally. 


\section{MODES OF PRODUCTION}

Furthermore, because rates of sharing did not clearly fluctuate in relation to the manipulations, it is difficult to discern which aspects of the manipulations contributed to sharing. Sharing may be attributable to the current conditions in which it occurred, or attributable to the fact that conditions were confounded with experimental history (i.e., there was no counterbalancing across dyads to control for order effects). Thus, although similarities among the dyads were found, the primary question of the study concerning controlling variables (i.e., what factors of resource production contribute to resource sharing) remains unanswered.

We believe a major contributor to the generally low rates of sharing observed in the current study relates to the discovery of a lower limit regarding modes of production and dyad maintenance (i.e., the prevention of restarts/token loss). Regardless of how many resources were harvested for any particular trial, the number of consecutive "double-zero" trials (i.e., a trial in which the number of resources harvested by both participants $=0$ ) could not exceed one less than the storage capacity. In other words, storage capacity $-1=$ maximum consecutive double-zero trials a group could sustain without having the participants lose tokens and the dyad restarting. This can be expressed as $\mathrm{SC}-1=\mathrm{DZT}$.

Since the storage capacity was two trials (i.e., the trial in which resources were received and the subsequent trial), the dyads could only sustain one doublezero trial. In every condition except the first, the dyads experienced two or more double-zero trials. Thus, it was nearly impossible to retain tokens outside of Condition 1, for Experiment 1, and nearly impossible to retain tokens for the entire experiment for Experiment 2. In short, outside of Condition 1, the participants never really had enough resources to retain tokens.

This problem is attributable to the simple fact that insufficient quantities of resources were used as independent variables in the study. The lack of available resources may also have contributed to the two unexpected consequences of "recipient-more" and "group-equal." It could be the case that such consequences arose in part due to extinction-induced variation resulting from the failure of sharing to retain tokens (see Lerman \& Iwata, 1996 for an overview of response characteristics during extinction). Future studies should make available more resources or increase the storage capacity beyond two trials to compensate for this unforeseen problem and further explore the factors contributing to the emergence of recipient-more and group-equal consequences.

It should also be further emphasized that the current study was a somewhat distant laboratory analogue of the hunter-gatherer phenomena described by Harris (1977, 1979). For instance, the "resources" weren't really food and building materials, and "survival" in no way meant the participants lost their lives. Furthermore, Experiment 1 allowed the participants to keep any tokens earned in 
previous conditions in the event of a restart, which raises questions related to the analogue nature of survival in the first experiment.

This issue concerns the fidelity or "realness" of the analogue and will be addressed two ways here. First, if resources are stimuli that acquire functions such that they are allocated or used in ways to gain certain reinforcers, then one may be able to say that the wooden sticks used in the current study served as functional resources. Survival, on the other hand, could take at least two forms: biological and economic. Functionally, failing to survive refers to conditions in which resource availability falls below resource requirements. Thus, one may be able to say that a primary difference between contingencies of survival in this study vs. the real-world is one concerning the magnitude of the consequences that are present when resource availability fails to meet resource requirements.

Brehmer (2005) provides a thoughtful analysis of such fidelity issues in "simulation" research and suggests that the degree of fidelity necessary in an experimental preparation is intimately linked to the goals of the particular study in question. For example, Brehmer describes computer simulations of forest fires that require participants to allocate resources in terms of manpower and equipment in relation to verbal information concerning wind direction and other common characteristics of forest fires. He then notes that such simulations can directly inform issues in complex real-world situations while being of a low fidelity because such studies are concerned with verbal decision-making responses in relation to the characteristics of a fire. Alternatively, Brehmer notes that simulations concerned with fine-motor skills, such as surgery, typically require a much higher degree of fidelity because the acquisition of such skills requires a good deal of direct experience with the task regardless of the verbal repertoire one may possess.

The current study was concerned with the allocation of resources in relation to resource availability. This study was hardly concerned with fine motor skills of the sort discussed by Brehmer (2005), and more closely resembles a study concerned with decision making in verbally sophisticated adults. Thus, fidelity issues may not be critical in the current type of research. However, another limitation of this study relates to verbal decision-making behavior. Although instructions were given to the participants regarding the experimental contingencies, and reference sheets summarizing the instructions were available for the participants to consult throughout the study (see Appendix A), no effort was made to assess the participants' ability to accurately state the contingencies operating during the study. It may be the case that the participants could not state the relevant contingencies operating in the study given the occurrence of, for instance, the "recipient-more" consequences. If this is indeed the case, future 


\section{MODES OF PRODUCTION}

researchers should consider the use of questionnaires to assess the degree to which the participants understand the rules stated at the beginning of the study that, if mastered, would allow participants to begin the session.

Finally, the current study was conducted using very low technology. Future studies should use a computerized apparatus. Such an apparatus could aid in the replicability of the methodology because confounds concerning the experimenter's body language and the immediacy with which the experimenter administers tokens or gives prompts (e.g., telling a participant they are "out") are always a possibility. Related to this is the fact that the experimenter and both participants were in close proximity to each other, which may introduce other confounds related to the bodily reactions of the dyad partner.

Sidman (1960) might regard the present study as one designed to "establish the existence of a behavioral phenomenon" (p. 23) which can expose phenomena drawn from the real-world to new types of experimental manipulations. In the present study, the new phenomena and manipulations concern the environmentbehavior relations between resource availability and resource allocation as conceptualized by Harris $(1977,1979)$.

\section{Integrating Existing Research}

At least one existing program of research has the potential to be integrated into the current methodology - optimal foraging research on energy-budgets (Pietras \& Hackenberg, 2001; Pietras, Cherek, Lane, \& Tcheremissine, 2006). For instance, recent optimal foraging work extending energy-budget research to individual (Pietras \& Hackenberg, 2001) and cooperative (Pietras et al., 2006) human behavior uses methods similar to those comprising survival contingencies in the current study. An energy-budget refers to the relation between: (a) an organism's current resource intake and reserves, and (b) an organism's resource requirements for survival over a given unit of time. When participants' earnings meet or exceed their resource requirement, those earnings are kept, and if earnings fall below their requirement, those earnings are lost. This characteristic is similar to the survival contingencies in the present study.

Furthermore, the Pietras et al. (2006) study asked a question similar to the present study concerning the combinations of resource availability and requirements that evoke cooperative or independent responding. Pietras et al. found that when average earnings for individual work fell below the energy requirement (i.e., participants couldn't keep many earnings by working individually), participants chose a cooperative resource-pooling response that produced stable and sufficient earnings. Furthermore, Pietras et al. extensively 
discussed their research in relation to anthropological field studies of food sharing, similar to the current study.

\section{Applications to Social Issues}

In the applied realm, the issues addressed in the current study may contribute to the more general evolution of an increasingly sophisticated cultural analysis of real-world events. Though cultural phenomena involve the interaction of a milieu of setting factors (Kantor, 1982), many phenomena seem to be intimately related to modes of production. We will now briefly discuss three recent events that could be dealt with more fully if a program of research in this area were to develop.

First, millions of Iraqi citizens have fled their homeland for Jordan and Syria since the beginning of the Iraq war in March of 2003 ("Nations Complain," 2007; "Numbers," 2007). Certainly, a major factor influencing such an exodus is the fact that an estimated 655,000 Iraqi civilian deaths between March 2003 and July 2006 are attributable to the U.S. led invasion (Byrne, 2006). However, Iraqis have also had to deal with disruptions in resource availability to the extent that the provision of basic resources such as water and power to many parts of the country are worse than prewar conditions (Buckley, 2006).

Modes of production could apply in a different way to help understand the difficulty of New Orleans' poor community (i.e., people with relatively inefficient modes of production) in recovering from hurricane Katrina. Interestingly, this difficulty of the poor was not likely a result of damage to exclusively poor neighborhoods. In areas that experienced light or no damage, $24.7 \%$ of the residents were classified as poor versus a slightly higher $29.2 \%$ in those areas with moderate to severe damage (Masozera, Baily, \& Kerchner, 2007; Logan, 2006). Thus, one could make the case that the majority of affected residents were not poor at all. According to Masozera, Baily, and Kerchner (2007) important factors responsible for the differential effects of the hurricane on the poor were a lack of private transportation to flee the area prior to the storm as well as a lack of insurance to deal with recovery after the storm. The former accounted for the more than 20,000 people who sought refuge in the Superdome (Center for Progressive Reform, 2005).

Lastly, disruptions in modes of production seemed to characterize the recent shutdown of "noncritical" state services in Pennsylvania due to a failure of the state government to approve its 2007-2008 budget ("Deal Ends," 2007). Because the government stalled, roughly 24,000 people employed in various state services including parks, highway maintenance, and licensing briefly went without pay. If such a disruption continued, Pennsylvania might have had to deal with a much 


\section{MODES OF PRODUCTION}

larger problem related to the failure of thousands to obtain the most basic resources needed for daily living such as food, gasoline, and rent.

\section{CONCLUSION}

This study may provide a general methodology for a program of research to systematize behavior analysis and cultural materialism. Some before-mentioned optimal foraging research (cf. Pietras, Cherek, Lane, \& Tcheremissine, 2006; Pietras \& Hackenberg, 2001) also claim to investigate aspects of survival in relation to resources. Even though the latter studies have important methodological differences concerning the ways in which resources are used, the general subject-matter is somewhat similar and warrants the consideration of a methodological fusion in future research.

While behavior analysts excel in studying behavior-environment relations, they have only begun to examine behavior in relation to resources and survival. Furthermore, the present study may be the first to attempt an experimental analysis of cultural materialism. Thus, to aid the future development of such research, we suggested areas in which the present methodology could come together with already existing programs of research to test the generality of behavioral principles under contingencies of survival. If the current study sparks a program of research, applications to real-world phenomena seem likely, and three present-day examples were given to illustrate the types of phenomena that become relevant to behavior analysts when resource-behavior relations are considered.

In closing, the most important offering of the current study resides in the potential for future research toward a more complex understanding of human behavior. What has not yet been fully appreciated among behavior analysts is the integral role of resources on the individual's daily life. As Ward (2008) suggests, "for the most part, the modern cultural environment in which behavior is nested cannot exist without some sort of income to 'purchase and maintain' that environment" (p. 22). Not only do we need resources for survival, which was the focus of the current study, but resources constitute the very stuff that comprise virtually every contextual condition in which the modern human behaves. It is in this way that Harris $(1977,1979)$ is able to place resources at the base of cultural development. At the infrastructural level, resources promote survival, but resources are also transformed into stimuli that take on endless forms such as the farmer's plow, the infant's crib, the artist's paintbrush, and the very words on this page. 


\section{WARD, EASTMAN, \& NINNESS}

\section{REFERENCES}

Brehmer, B. (2005). Micro-worlds and the circular relation between people and their environment. Theoretical Issues in Ergonomics Science, 6, 73-93. http://dx.doi.org/10.1080/14639220512331311580

Buckley, B. (2006). Infrastructure work starved by loss of funds to security. Engineering NewsRecord, 256, 16.

Byrne, R. (2006). New study of Iraq's civilian deaths confirms earlier estimates. Chronicle of Higher Education, 53, 23.

Center for Progressive Reform, (2005). An Unnatural Disaster: The Aftermath of Hurricane Katrina. Report. CPR Publication, 512.

Deal ends Pennsylvania government's partial shutdown. (2007, July 9). Retrieved July 9, 2007, from http://www.cnn.com/2007/POLITICS/07/09/pennsylvania.shutdown.ap/index.html

Glenn, S. S. (1988). Contingencies and metacontingencies: Toward a synthesis of behavior analysis and cultural materialism. The Behavior Analyst, 11, 161-179.

Glenn, S. S. (2003). Operant contingencies and the origin of cultures. In Lattal, K. A., \& Chase, P. N. (Eds.), Behavior theory and philosophy (pp. 223-242). New York: Kluwer Academic/Plenum Publishers.

Harris, M. (1977). Cannibals and kings: The origins of cultures. New York: Random House.

Harris, M. (1979). Cultural materialism: The struggle for a science of culture. New York: Random House.

Kantor, J. R. (1982). Cultural Psychology. Chicago, IL: Principia Press.

Lerman, D. C., \& Iwata, B. A. (1996). Developing a technology for the use of operant extinction in clinical settings: An examination of basic and applied research. Journal of Applied Behavior Analysis, 29, 345-382. http://dx.doi.org/10.1901/jaba.1996.29-345

Lloyd, K. E. (1985). Behavioral Anthropology: A review of Marvin Harris' Cultural materialism. Journal of the Experimental Analysis of Behavior, 43, 279-287. http://dx.doi.org/10.1901/jeab.1985.43-279

Logan, J.R. (2006). The impact of Katrina: Race and class in storm-damaged neighborhoods. S4, Spatial structures in the social sciences, hurricane Katrina project. Providence, R.I.: Brown University. Retrieved August 2, 2007 from http://www.s4.brown.edu/Katrina/report.pdf.

Malagodi, E. F. (1986). On radicalizing behaviorism: A call for cultural analysis. The Behavior Analyst, 9, 1-17.

Malagodi, E. F., \& Jackson, K. (1989). Behavior analysis and cultural analysis: Troubles and issues. The Behavior Analyst, 12, 17-33.

Malott, R. W. (1988). Rule-governed behavior and behavioral anthropology. The Behavior Analyst, 11, 181-203.

Masozera, M., Bailey, M., \& Kerchner, C. (2007). Distribution of impacts of natural disasters across income groups: A case study of New Orleans. Ecological Economics, 63, 299-306. http://dx.doi.org/10.1016/j.ecolecon.2006.06.013

Nations complain about influx of Iraq refugees. (2007, July 26). Retrieved July 27, 2007, from http://www.msnbc.msn.com/id/19980375/

Numbers. (2007, April 9). Time, 169, 18.

Pietras, C. J., Cherek, D. R., Lane, S. D., Tcheremissine, O. (2006). Risk reduction and resource pooling on a cooperation task. Psychological Record, 56, 387-410.

Pietras, C. J., \& Hackenberg, T. D. (2001). Risk-sensitive choice in humans as a function of an earnings budget. Journal of the Experimental Analysis of Behavior, 76, 1-19. http://dx.doi.org/10.1901/jeab.2001.76-1

Sidman, M. (1960). Tactics of scientific research. Boston, MA: Authors Cooperative, Inc.

Skinner, B. F. (1953). Science and human behavior. New York: Free Press.

Skinner, B. F. (1981). Selection by consequences. Science, 213, 501-504. http://dx.doi.org/10.1126/science.7244649 
Vargas, E. A. (1985). Cultural contingencies: A review of Marvin Harris's Cannibals and Kings. Journal of the Experimental Analysis of Behavior, 43, 419-428.

Ward, T. A. (2008). Gary Becker and behavior analysis: Collaboration for complex human behavior. Association for Behavior Analysis International Newsletter, 31 (2), 21-22.

\section{ApPendix A: The Reference SHEeT}

\section{What you can do}

First task each trial:

- 1 stick in P. Req. = do anything.

- No stick in P. Req. = lose everything in condition, and "out" until the same happens to the other participant.

- Resets to zero every trial.

With extra sticks:

- 5 sticks in Bank = 1 bead. Cannot remove anything from Bank.

- Store sticks in "store" for max. of two trials. You can move sticks from here to P. Req., Bank, or give to other participant.

- Can give sticks to the other participant.

\section{APPENDIX B: THE INSTRUCTIONS}

\section{The Instructions}

In this experiment, you will have the opportunity to win a drawing for a $\$ 25$ gas card $<<$ show card $>>$ by earning plastic beads $<<$ point to beads $>>$. Your name will be entered once for every bead you keep at the end of the experiment; so the more beads you have, the more likely you will win. You will be notified if you win at the end of the entire study.

You will receive a bead for every five sticks placed in your box labeled "bank" $<<$ point to banks $>>$. At the beginning of each trial, one of you will draw a card indicating how much and what kind of sticks each of you will receive that trial.

The first thing you have to do each trial is place a stick into your box labeled "participation requirement" $<<$ point to p. requirement $>>$. This enables you to do anything else during that trial and resets to zero every trial. If this is not maintained, then you lose everything earned in that condition and cannot continue until the same happens to the other participant. 
If you maintain your participation requirement for that trial, you can do three things. First, you can put extra sticks into your bank to earn tokens. Once a stick is here it can't be removed $<<$ pause for a second $>>$. Second, you can store extra sticks into your box labeled "store" for two trials. You can move sticks from the store to the participation requirement or bank at any time $<<$ pause for a second $>>$. And, third, you can give extra sticks to the other participant.

You $<<$ person to my right $>>$ will be participant one so you draw the amount listed under P1 and you $<<$ person to my left $>>$ will be participant two, so you will draw the amount listed under P2.

Any questions?

Once we begin, there will be no talking. Unless you have a specific question about the cards, all other questions can be answered on your sheets. 